\title{
REPORT
}

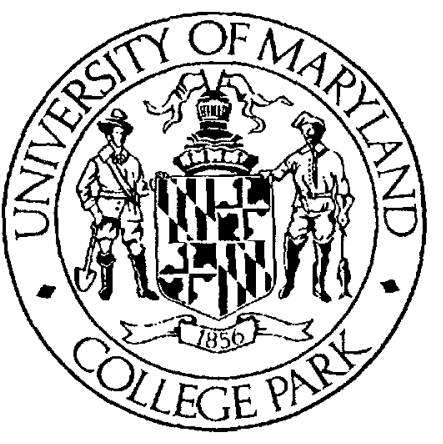

\begin{tabular}{lllllll}
\hline$S$ & $Y$ & $S$ & $T$ & $E$ & $M$ & $S$
\end{tabular}

R E S E A R C

\begin{tabular}{llllll}
$C$ & $E$ & $N$ & $T$ & $E$ & $R$ \\
\hline
\end{tabular}

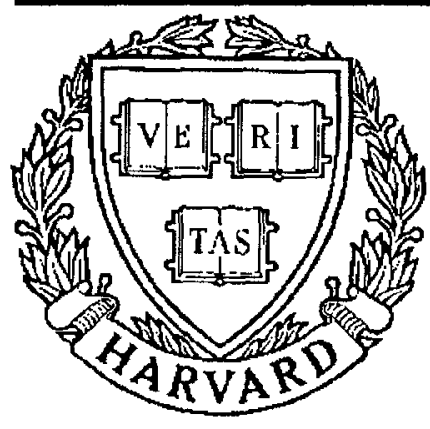

Supported by the

National Science Foundation

Engineering Research Center

Program (NSFD CD 8803012), Industry and the University

\section{On the Dynamics of Floating Four-Bar Linkages}

\author{
by R. Yang and P.S. Krishnaprasad
}




\title{
ON THE DYNAMICS OF FLOATING FOUR-BAR LINKAGES *
}

\author{
Rui Yang \\ P. S. Krishnaprasad
}

\author{
Electrical Engineering Department \\ $\&$ \\ Systems Research Center \\ University of Maryland, College Park.
}

\begin{abstract}
The hamiltonian structure of floating, planar four-bar linkages is discussed. The geometry of configuration space is related to the classical theory of mechanisms due to Grashof. For generic value of kinematic parameters, the techniques of symplectic (and Poisson) reduction apply.
\end{abstract}

\section{INTRODUCTION}

There has been significant progress in our understanding of the hamiltonian structure of serial-link (or open chain) multibody systems $\left[2,3,9,11,12,15^{-18,21-23}\right]$. The use of geometric methods, symmetry principles and reduction has led to deeper knowledge of the phase portraits of model problems. This insight has been helpful in developing appropriate control-theoretic tools. The primary source of motivation for these problems has been

* This work was supported in part by the AFOSR University Research Initiative Program under grant AFOSR-87-0073 and by the National Science foundation's Engineering Research Center Program: NSFD CDR 8803012. 
in acrospace engineering where imaginative designs of multibody spacecraft have been proposed and on occasion realized $[4,5,24]$.

In contrast, multibody systems with kinematic loops present serious challenges. The loop constraint may lead to singular configuration spaces. The Dirac theory of constraints $[6,20]$ applies in the smooth setting. Little or nothing is known about hamiltonian structure and phase portraits in concrete cases. However, engineering applications suggest that multibody systems with kinematic loops are of practical importance [10]. Parallel linkage based robot manipulators are contemplated for space applications.

Here we discuss the geometry and dynamics of floating four-bar linkages. In this model problem, the classical Grashof criterion [8,10,14] appears through conditions for the configuration space to be a smooth manifold. The topology of the configuration space is also related to the Grashof criteria. We explore symmetry properties, hamiltonian structure and reduction of four bar linkage dynamics. Explicit computation of constrained dynamics is difficult. Yet in the present setting, using geometric techniques, one can infer qualitative properties without recourse to explicit analytic representation of the constrained dynamics. We use a theorem of Smale to determine relative equilibria for four-bar linkage dynamics.

\section{NOTATIONS \& GEOMETRIC CONSTRAINTS}

The structure of a closed floating four-bar linkage is represented in Fig. 1. The bars are labeled clockwise from 0 to 3 as shown. We define the following quantities.

$\mathbf{d}_{i j} \quad$ the vector of hinge point which connects $i$-th bar with $j$-th bar relative to a body-fixed frame with origin at the center of mass of the $i$-th body;

$\mathbf{r}_{i} \quad$ the position vector of the center of mass of $i$-th bar relative to an inertial observer;

$\mathbf{r}_{i}^{c} \quad$ the vector from the system center of mass to the center of mass of $i$-th bar; 


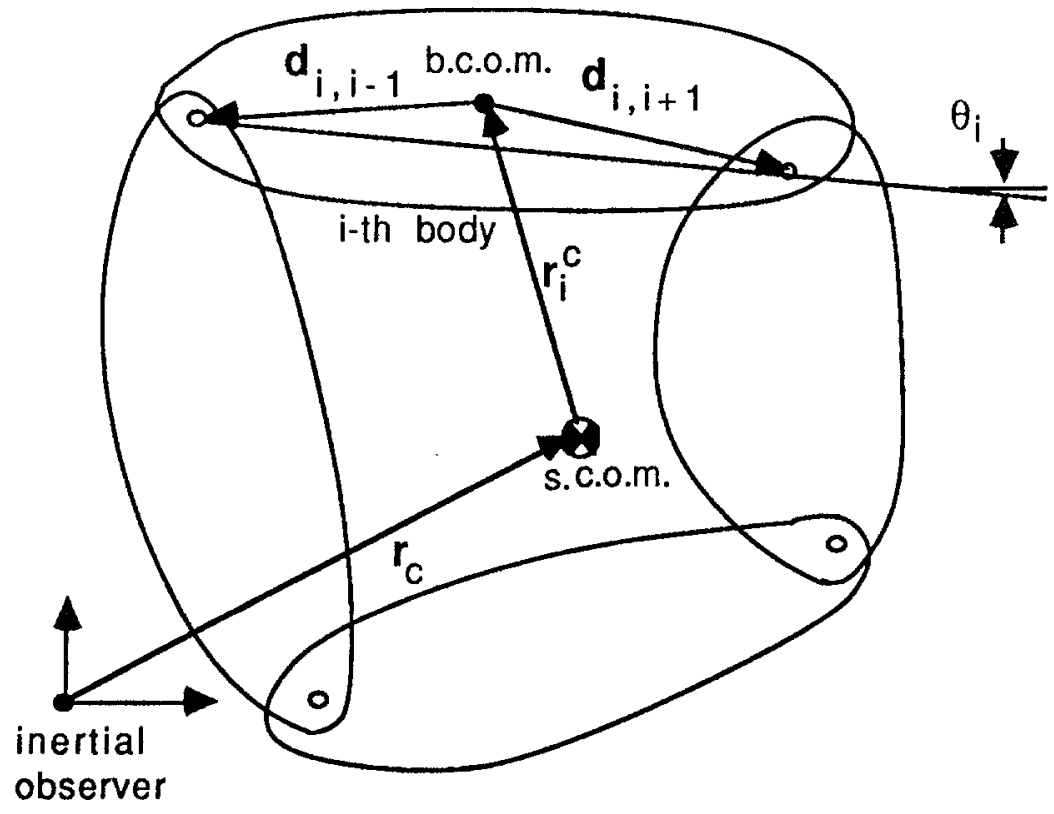

Fig. 1 The general structure of four-bar linkage

$\mathbf{r}_{c}$ the position of the system center of mass relative to the reference point of the inertial observer;

$R\left(\theta_{i}\right) \quad$ the rotation through angle $\theta_{i}$ giving the orientation of $i$-th bar relative to the inertial space;

$$
R\left(\theta_{i}\right)=\left(\begin{array}{cc}
\cos \left(\theta_{i}\right) & -\sin \left(\theta_{i}\right) \\
\sin \left(\theta_{i}\right) & \cos \left(\theta_{i}\right)
\end{array}\right)
$$

$R\left(\theta_{i j}\right) \quad$ joint rotation between $i$-th and $j$-th bar,

$$
R\left(\theta_{i j}\right)=R\left(\theta_{i}-\theta_{j}\right)=R\left(\theta_{i}\right) R\left(-\theta_{j}\right)
$$

$l_{i}$

the distance between the joints on $i$-th bar, (or "length" of $i$-th bar); all $l_{i}>0 ; l_{i}=\left\|\mathrm{d}_{i, i+1}-\mathrm{d}_{i, i-1}\right\| ;$ 


$$
m=\sum_{i=0}^{3} m_{i}
$$

With the above notations, any pair of adjacent bodies is connected by the following relation, so called hinge constraint,

$$
\mathbf{r}_{i+1}^{c}=\mathbf{r}_{i}^{c}+R\left(\theta_{i}\right) \mathbf{d}_{i, i+1}-R\left(\theta_{i+1}\right) \mathbf{d}_{i+1, i} \quad i=0,1,2,3(\bmod 4) .
$$

By eliminating $\mathbf{r}_{i}^{c}$ in Eq.(2.1) we find the loop constraint or closure constraint,

$$
\sum_{i=0}^{3} R\left(\theta_{i}\right)\left(\mathbf{d}_{i, i+1}-\mathrm{d}_{i, i-1}\right)=0
$$

where we adopt the convention that $d_{i, 4}=d_{i, 0}$ and $d_{4, i}=d_{0, i}$.

\section{THE CONFIGURATION SPACE}

In this section we investigate the conditions under which the loop constraint, Eq.(2.2), describes a submanifold with respect to the configuration manifold of an open four-bar chain.

For a planar floating four-bar open chain, the configuration space is

$$
M=R^{2} \times S^{1} \times S^{1} \times S^{1} \times S^{1},
$$

where $M$ is a 6 -dimensional smooth manifold with local coordinates of the form,

$$
\mathbf{q}=\left(x_{0}, y_{0}, \theta_{0}, \theta_{1}, \theta_{2}, \theta_{3}\right)
$$

This corresponds to keeping track of a material point (say center of mass on one of the bodies) and the four absolute orientations. See $[12,21,22]$ for the hamiltonian mechanics of such open chains. 
For a closed four bar mechanism as considered in this paper, the configuration space denoted by $Q$ is a subset of $M$ determined by Eq.(2.2), or simply

$$
Q=\left\{\mathbf{q} \in M \mid \mathbf{F}(\mathbf{q})=\sum_{i=0}^{3} R\left(\theta_{i}\right)\left(\mathbf{d}_{i, i+1}-\mathbf{d}_{i, i-1}\right)=0\right\} .
$$

Note that $\mathbf{F}: M \longrightarrow R^{2}$, and from [1] we know that if $\mathbf{0}$ is a regular value of $\mathbf{F}$, i.e. $\partial \mathbf{F} / \partial \mathbf{q}$ has full rank for all $\mathbf{q} \in Q$, then $Q$ is a submanifold of $M$.

From (3.1) we have,

$$
\frac{\partial \mathbf{F}}{\partial \mathbf{q}}=\left(\begin{array}{cccccc}
0 & 0 & -l_{0} \sin \left(\theta_{0}\right) & -l_{1} \sin \left(\theta_{1}\right) & -l_{2} \sin \left(\theta_{2}\right) & -l_{3} \sin \left(\theta_{3}\right) \\
0 & 0 & l_{0} \cos \left(\theta_{0}\right) & l_{1} \cos \left(\theta_{1}\right) & l_{2} \cos \left(\theta_{2}\right) & l_{3} \cos \left(\theta_{3}\right)
\end{array}\right)
$$

Then it is easy to check that all the nontrivial determinants of $2 \times 2$ submatrices are given by the set of expressions,

$$
\begin{aligned}
& g_{1}(\mathbf{q})=l_{0} l_{1} \sin \left(\theta_{1}-\theta_{0}\right) \\
& g_{2}(\mathbf{q})=l_{0} l_{2} \sin \left(\theta_{2}-\theta_{0}\right) \\
& g_{3}(\mathbf{q})=l_{0} l_{3} \sin \left(\theta_{3}-\theta_{0}\right) \\
& g_{4}(\mathbf{q})=l_{1} l_{2} \sin \left(\theta_{2}-\theta_{1}\right) \\
& g_{5}(\mathbf{q})=l_{1} l_{3} \sin \left(\theta_{3}-\theta_{1}\right) \\
& g_{6}(\mathbf{q})=l_{2} l_{3} \sin \left(\theta_{3}-\theta_{2}\right) .
\end{aligned}
$$

Therefore for each $\mathbf{q} \in Q$, if there exists an $i$ such that $g_{i}(\mathbf{q}) \neq 0, Q$ is a submanifold of $M$.

It is obvious that the above condition depends on the lengths of the links. To find the condition on the links, we can use an equivalent way, that is, find necessary conditions on the lengths of the links such that $g_{i}(\mathbf{q})=0$ for all $i$.

From Eqs.(3.2) it can be seen that if

$$
\theta_{1}-\theta_{0}=0 \text { or } \pi
$$

and

$$
\theta_{3}-\theta_{2}=0 \text { or } \pi
$$


and

$$
\theta_{3}-\theta_{0}=0 \text { or } \pi
$$

then $g_{i}(\mathbf{q})=0$ for all $i$.

Premultiplying Eq.(2.2) by $R\left(\theta_{0}\right)$, we get following equivalent closure constraint equations

$$
\begin{gathered}
l_{0}+l_{1} \cos \left(\theta_{1}-\theta_{0}\right)+l_{2}\left(\cos \left(\theta_{3}-\theta_{2}\right) \cos \left(\theta_{3}-\theta_{0}\right)+\sin \left(\theta_{3}-\theta_{2}\right) \sin \left(\theta_{3}-\theta_{0}\right)\right) \\
+l_{3} \cos \left(\theta_{3}-\theta_{2}\right)=0 \\
l_{1} \sin \left(\theta_{1}-\theta_{0}\right)+l_{2}\left(\cos \left(\theta_{3}-\theta_{2}\right) \sin \left(\theta_{3}-\theta_{0}\right)-\sin \left(\theta_{3}-\theta_{2}\right) \cos \left(\theta_{3}-\theta_{0}\right)\right) \\
+l_{3} \sin \left(\theta_{3}-\theta_{2}\right)=0
\end{gathered}
$$

The conditions given in Eq.(3.3) make Eq.(3.4) $b$ trivial. By choosing all the possible value of $\theta_{1}-\theta_{0}, \theta_{3}-\theta_{2}$ and $\theta_{3}-\theta_{0}$ given in Eq.(3.3), from Eq.(3.4) $a$, we can find the link conditions, which are summarized in Table 1.

Theorem 3.1: If $l_{0} \pm l_{1} \pm l_{2} \pm l_{3} \neq 0, Q$ is a submanifold of $M$.

From Table 1 , it is easy to observe that case (i) can never happen since $l_{i}$ are assumed to be positive. In addition, cases (iii), (iv), (v) and (vi) are trivial cases since none of them can be formed by any general four-bar closed loop. In these cases the configuration spaces lose one degree of freedom and are three dimensional.

Furthermore, Theorem 3.1 can be simplified by ignoring the labels on the bars. To do this, we first recall some definitions and results in the classical theory of mechanisms $[8,13,14]$. We define following quantities

$$
\begin{aligned}
s & =\text { length of shortest bar } \\
l & =\text { length of longest bar } \\
p, q & =\text { lengths of intermediate bars. }
\end{aligned}
$$

A link which is free to rotate through $2 \pi$ with respect to a second link is said to revolve relative to the second bar and is referred to as a crank. Any bar which does not revolve is called a rocker. If it is possible for all bars to become simultaneously aligned, such a state is called a change point and the linkage is said to be a change-point mechanism. 


\section{Table 1.}

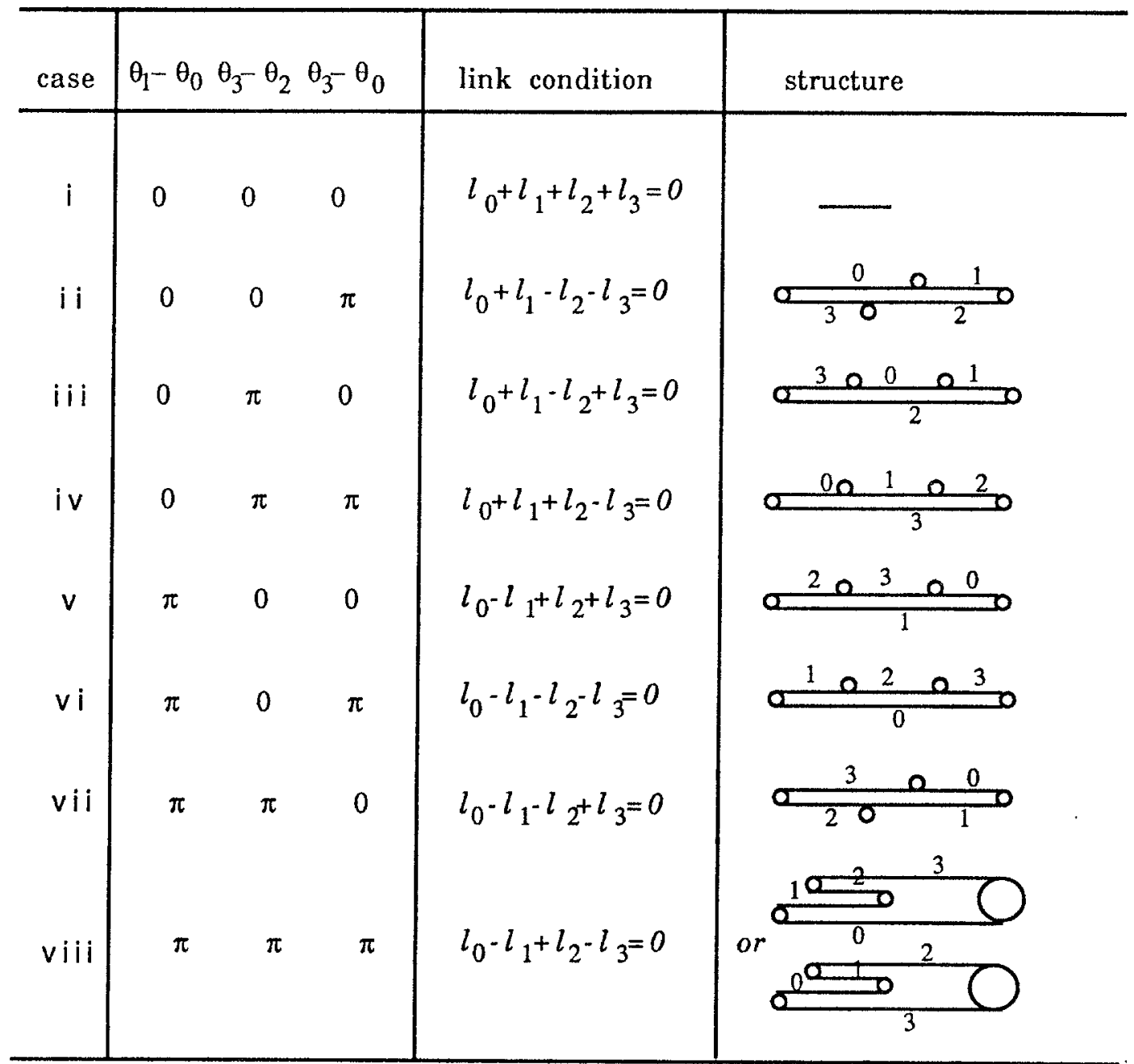

Theorem 3.2:(Grashof) (1) A four-bar mechanism has at least one revolving link if

$$
s+l \leq p+q
$$

and all three will rock if

$$
s+l>p+q .
$$

(2) A four-bar mechanism is a change-point mechanism iff

$$
s+l=p+q .
$$

Remark: It is easy to check that the cases (ii), (vii) and (viii) in Table 1. correspond to $s+l=p+q$ i.e. they correspond to change-point mechanisms. 
Corollary 3.3: If $l \leq s+q+p$ and $s+l \neq p+q, Q$ is a submanifold of $M$.

Remark: Note that if $l>s+q+p$, the mechanism is not constructible.

In order to find a topological description of the configuration manifold $Q$, we first introduce following fact.

Proposition 3.4: If $s+l<p+q$ and $l_{1}=s$, i.e. $l_{1}=\min _{0 \leq i \leq 3}\left(l_{i}\right)$, then $\theta_{3}-\theta_{2} \not \equiv k \pi$ for $k \in Z$.

Proof: The mechanism can be assembled with $s$ adjacent to $l$ or with $s$ opposite $l$. And, $l$ can be $l_{0}, l_{2}$ or $l_{3}$. If $\theta_{3}-\theta_{2}=k \pi$, the whole structure attains a triangular shape which has the property that the sum of two sides is larger than the third one. Then it is easy to check that all possible cases will lead to

$$
s+l>p+q
$$

This is a contradiction.

From Grashof's theorem and the above proposition we can get a topological description for $Q$.

Corollary 3.5: For a crank mechanism with $s+l<p+q$, each connected component is diffeomorphic to

$$
Q=R^{2} \times S^{1} \times S^{1}
$$

with parametrization $\left(x_{0}, y_{0}, \theta_{0}, \theta_{1}\right)$ if $l_{1}=s$ is assumed.

Proof: We just need to prove that $\theta_{2}$ and $\theta_{3}$ can be uniquely determined by $\theta_{0}$ and $\theta_{1}$. The constraints given in Eq.(3.1) give the relations among $\theta_{i}$ 's and they have continuous partial derivatives with respect to $\theta_{i}$. Since

$$
\operatorname{det}\left(\frac{\partial \mathrm{F}}{\partial\left(\theta_{2}, \theta_{3}\right)}\right)=l_{2} l_{3} \sin \left(\theta_{3}-\theta_{2}\right)
$$

it follows from the Implicit Function Theorem that if

$$
\theta_{3}-\theta_{2} \neq k \pi \quad \text { for } k \in Z
$$

in some neighborhood of a point $\left(\theta_{0}, \theta_{1}, \theta_{2}, \theta_{3}\right)$, then there exists a unique pair of functions $f_{1}$ and $f_{2}$ such that

$$
\theta_{2}=f_{1}\left(\theta_{0}, \theta_{1}\right) \text { and } \theta_{3}=f_{2}\left(\theta_{0}, \theta_{1}\right)
$$


and

$$
\mathbf{F}\left(\theta_{0}, \theta_{1}, f_{1}\left(\theta_{0}, \theta_{1}\right), f_{2}\left(\theta_{0}, \theta_{1}\right)\right)=0
$$

Using the result in above proposition, the proof is complete. In addition, as seen from [13], there exists a pair of function $\hat{f}_{1}$ and $\hat{f}_{2}$ such that

$$
\theta_{2}=\theta_{0}+\hat{f}_{1}\left(\theta_{1}-\theta_{0}\right) \text { and } \theta_{3}=\theta_{0}+\hat{f}_{2}\left(\theta_{1}-\theta_{0}\right) \text {. }
$$

Remark: Following Gibson and Newstead [7] one can show that if $l+s<p+q$, then there are two connected components of $Q$ and if $s+l>p+q$, the space $Q$ is connected and diffeomorphic to $R^{2} \times S^{1} \times S^{1}$.

Since the configuration space under the condition given in Corollary 3.5 has an explicit parametrization, in the rest of this paper we shall study the system on this space mainly.

\section{KINETIC ENERGY}

In this section we shall derive the kinetic energy, or Lagrangian since we assumed that no potential energy is involved, for the whole system. The basic idea is to write the kinetic energy for each individual body first and then use the constraint equations to eliminate extra variables.

The kinetic energy of the $i$-th bar is

$$
T_{i}=\frac{1}{2} \omega_{i}^{2} I_{i}+\frac{1}{2} m_{i}\left\|\dot{\mathbf{r}}_{i}\right\|^{2}
$$

where $\omega_{i}=\dot{\theta}_{i}$. The total kinetic energy is

$$
T=\frac{1}{2} \sum_{i=0}^{3} \omega_{i}^{2} I_{i}+\frac{1}{2} \sum_{i=0}^{3} m_{i}\left\|\dot{\mathbf{r}}_{i}\right\|^{2} .
$$

To describe the kinetic energy relative to the center of mass, we have following useful equations,

$$
\begin{aligned}
\mathbf{r}_{i} & =\mathbf{r}_{c}+\mathbf{r}_{i}^{c} \quad i=0,1,2,3 \\
\sum_{i=0}^{3} m_{i} \mathbf{r}_{i}^{c} & =0
\end{aligned}
$$


By applying Eqs.(4.2) and (4.3), Eq.(4.1) becomes,

$$
T=\frac{1}{2} \sum_{i=0}^{3} \omega_{i}^{2} I_{i}+\frac{1}{2} \sum_{i=0}^{3} m_{i}\left\|\dot{\mathbf{r}}_{i}^{c}\right\|^{2}+\frac{1}{2} m\left\|\dot{\mathbf{r}}_{c}\right\|^{2} .
$$

Applying Eq.(2.1) and Eq.(4.2), we get

$$
\begin{aligned}
\mathbf{r}_{i}^{c}=\frac{1}{m} & {\left[R\left(\theta_{i-1}\right) m_{i-1} \mathbf{d}_{i-1, i}\right.} \\
& -R\left(\theta_{i}\right)\left(m_{i-1} \mathbf{d}_{i, i-1}+\left(m_{i+1}+m_{i+2}\right) \mathrm{d}_{i, i+1}\right) \\
& +R\left(\theta_{i+1}\right)\left(\left(m_{i+1}+m_{i+2}\right) \mathbf{d}_{i+1, i}-m_{i+2} \mathbf{d}_{i+1, i+2}\right) \\
& \left.+R\left(\theta_{i+2}\right) m_{i+2} \mathbf{d}_{i+2, i+1}\right]
\end{aligned}
$$

for $i=0,1,2,3(\bmod 4)$. Furthermore,

$$
\begin{aligned}
\dot{\mathbf{r}}_{i}^{c}=\frac{1}{m} & {\left[m_{i-1} \hat{\omega}_{i-1} R\left(\theta_{i-1}\right) \mathbf{d}_{i-1, i}\right.} \\
& -\hat{\omega}_{i} R\left(\theta_{i}\right)\left(m_{i-1} \mathbf{d}_{i, i-1}+\left(m_{i+1}+m_{i+2}\right) \mathbf{d}_{i, i+1}\right) \\
& +\hat{\omega}_{i+1} R\left(\theta_{i+1}\right)\left(\left(m_{i+1}+m_{i+2}\right) \mathbf{d}_{i+1, i}-m_{i+2} \mathbf{d}_{i+1, i+2}\right) \\
& \left.+\hat{\omega}_{i+2} R\left(\theta_{i+2}\right) m_{i+2} \mathbf{d}_{i+2, i+1}\right]
\end{aligned}
$$

for $i=0,1,2,3(\bmod 4)$, where

$$
\hat{\omega}_{i}=\left(\begin{array}{cc}
0 & -\omega_{i} \\
\omega_{i} & 0
\end{array}\right) .
$$

By substituting the formula for $\dot{\mathbf{r}}_{i}^{c}$ into Eq.(4.4), we get a more compact expression for the kinetic energy

$$
T=\frac{1}{2}<\tilde{\omega}, \tilde{\mathbf{J}} \tilde{\omega}>+\frac{1}{2} m\left\|\dot{\mathbf{r}}_{c}\right\|^{2}
$$

where $\tilde{\omega}=\left(\omega_{0}, \omega_{1}, \omega_{2}, \omega_{3}\right)^{T}$ and $\tilde{\mathbf{J}}=\left(\tilde{J}_{i, j}, i, j=0,1,2,3\right)$ is a $4 \times 4$ symmetric matrix, with elements given as follows.

Let

$$
\begin{aligned}
M_{i}^{I}= & \frac{1}{m^{2}}\left[m_{i} m_{i+1}\left(m_{i}+m_{i+1}\right)\right. \\
& +m_{i+1}\left(m_{i+1} m_{i-1}+m_{i} m_{i+2}\right) \\
& \left.+m_{i} m_{i+2}\left(m_{i+1}+m_{i+2}\right)\right] \\
M_{i}^{I I}= & \frac{m_{i}}{m^{2}}\left(m_{i+2}^{2}-m_{i+1} m_{i-1}\right) \\
M_{i}^{I I I}= & \frac{m_{i} m_{i+2}}{m^{2}}\left(m_{i+1}+m_{i-1}\right) .
\end{aligned}
$$


Then

$$
\begin{aligned}
\tilde{J}_{i i}= & I_{i}+M_{i}^{I}\|\mathrm{~d}\|_{i, i+1}^{2}+M_{i-1}^{I}\|\mathrm{~d}\|_{i, i-1}^{2} \\
& -2 M_{i-1}^{I I}<\mathrm{d}_{i, i+1}, \mathrm{~d}_{i, i-1}> \\
\tilde{J}_{i, i+1}= & -M_{i}^{I}<\mathrm{d}_{i, i+1}, R\left(\theta_{i+1, i}\right) \mathrm{d}_{i+1, i}> \\
& +M_{i}^{I I}<\mathrm{d}_{i, i+1}, R\left(\theta_{i+1, i}\right) \mathrm{d}_{i+1, i+2}> \\
& +M_{i-1}^{I I}<\mathrm{d}_{i, i-1}, R\left(\theta_{i+1, i}\right) \mathrm{d}_{i+1, i}> \\
& +M_{i}^{I I I}<\mathrm{d}_{i, i-1}, R\left(\theta_{i+1, i}\right) \mathrm{d}_{i+1, i+2}> \\
\tilde{J}_{i, i+2}= & -M_{i}^{I I}<\mathrm{d}_{i, i+1}, R\left(\theta_{i+2, i}\right) \mathrm{d}_{i+2, i+1}> \\
& -M_{i+1}^{I I I}<\mathrm{d}_{i, i+1}, R\left(\theta_{i+2, i}\right) \mathrm{d}_{i+2, i-1}> \\
& -M_{i+2}^{I I}<\mathrm{d}_{i, i-1}, R\left(\theta_{i+2, i}\right) \mathrm{d}_{i+2, i-1}> \\
& -M_{i}^{I I I}<\mathrm{d}_{i, i-1}, R\left(\theta_{i+2, i}\right) \mathrm{d}_{i+2, i+1}>
\end{aligned}
$$

for $i=0,1,2,3(\bmod 4)$.

Up to now, we have not applied the condition on the lengths of links of a closed loop. It is clear that, in Eq.(4.6), $\theta_{i}$ and the velocities $\omega_{i}, i=0,1,2,3$ are involved. Since the chain is closed, these variables are not independent. As shown in the proof of Corollary 3.5, if $s+l<p+q$ and $l_{1}=s$, we have an one-to-one map from $\left(\theta_{0}, \theta_{1}\right)$ to $\left(\theta_{2}, \theta_{3}\right)$. Therefore every element of the matrix $\tilde{\mathbf{J}}$ can be expressed as a function of $\theta_{1}-\theta_{0}$ uniquely.

Under the same conditions on links, i.e. $s+l<p+q$, the loop constraints (2.2) yield a relation between $\left(\omega_{0}, \omega_{1}\right)$ and $\left(\omega_{2}, \omega_{3}\right)$ :

$$
\left(\begin{array}{l}
\omega_{2} \\
\omega_{3}
\end{array}\right)=\Omega\left(\begin{array}{l}
\omega_{0} \\
\omega_{1}
\end{array}\right)
$$

where

$$
\Omega=\left(\begin{array}{cc}
-\frac{l_{0} \sin \left(\theta_{3}-\theta_{0}\right)}{l_{2} \sin \left(\theta_{3}-\theta_{2}\right)} & -\frac{l_{1} \sin \left(\theta_{3}-\theta_{1}\right)}{l_{2} \sin \left(\theta_{3}-\theta_{2}\right)} \\
\frac{l_{0} \sin \left(\theta_{2}-\theta_{0}\right)}{l_{3} \sin \left(\theta_{3}-\theta_{2}\right)} & \frac{l_{1} \sin \left(\theta_{2}-\theta_{1}\right)}{l_{3} \sin \left(\theta_{3}-\theta_{2}\right)}
\end{array}\right) .
$$

Here, the matrix $\Omega$ is well defined because of the result in Proposition 3.4. Again, since $s+l<p+q$ and $s=l_{1}$, elements of matrix $\Omega$ are functions of $\theta_{1}-\theta_{0}$.

We summarize the above discussion in the following theorem. 
Theorem 4.1: If $s+l<p+q$ and $l_{1}=s$, the kinetic energy, or Lagrangian, can be represented as

$$
T=L=\frac{1}{2}<\omega, \mathbf{J} \omega>+\frac{1}{2} m\left\|\dot{\mathbf{r}}_{c}\right\|^{2}
$$

where $\omega=\left(\omega_{0}, \omega_{1}\right)^{T}$ and

$$
\mathbf{J}=\left(\begin{array}{ll}
I & \Omega^{T}
\end{array}\right) \tilde{\mathbf{J}}\left(\begin{array}{l}
I \\
\Omega
\end{array}\right)
$$

for $\tilde{\mathrm{J}}$ given in (4.6)-(4.8) and $\Omega$ given in (4.9). In addition, the elements of $\mathrm{J}$ are the functions of $\left(\theta_{1}-\theta_{0}\right)$.

Before ending this section, we give a property of the matrix $\Omega$ which will be used in section 6 .

Proposition 4.2: Under the assumptions of Theorem 4.1,

$$
\left(\begin{array}{l}
1 \\
1
\end{array}\right)=\Omega\left(\begin{array}{l}
1 \\
1
\end{array}\right) \text {. }
$$

Proof: Premultiplying Eq.(2.2) by $R\left(\theta_{2}\right)$ and $R\left(\theta_{3}\right)$, we get

$$
l_{0} \sin \left(\theta_{0}-\theta_{2}\right)+l_{1} \sin \left(\theta_{1}-\theta_{2}\right)+l_{3} \sin \left(\theta_{3}-\theta_{2}\right)=0
$$

and

$$
l_{0} \sin \left(\theta_{0}-\theta_{3}\right)+l_{1} \sin \left(\theta_{1}-\theta_{3}\right)+l_{2} \sin \left(\theta_{2}-\theta_{3}\right)=0
$$

respectively. They immediately imply the claim.

\section{SYMMETRY AND INTEGRAL}

We shall show here that a floating four-bar linkage is a simple mechanical system with symmetry in the sense of Smale $[1,19]$.

A simple mechanical system with symmetry is a 4-tuple $(Q, K, V, G)$, where,

(i) $(Q, K)$ is a Riemannian configuration manifold with metric $K$;

(ii) $G$ is a Lie group acting on $Q$ on the left,

$$
\begin{aligned}
\Phi: G \times Q & \rightarrow Q \\
(g, q) & \mapsto \Phi_{g}(q) \triangleq \Phi(g, q)
\end{aligned}
$$


such that for each $g \in G, \Phi_{g}$ is an isometry of $(Q, K)$;

(iii) $V: Q \rightarrow R$ is a G-invariant potential function.

The associated Lagrangian is defined by

$$
\begin{aligned}
L: T Q & \rightarrow R \\
v_{q} & \mapsto L\left(v_{q}\right)=\frac{1}{2} K\left(v_{q}, v_{q}\right)-V \circ \tau\left(v_{q}\right)
\end{aligned}
$$

where $\tau: T Q \rightarrow Q$ is the canonical tangent projection. The Legendre transform $F L$ of $L$ is given here by the vector bundle isomorphism

$$
K^{b}: T Q \rightarrow T^{*} Q
$$

satisfying

$$
K^{b}\left(v_{q}\right) \cdot w_{q}=K\left(v_{q}, w_{q}\right) \quad \forall v_{q}, w_{q} \in T_{q} Q .
$$

The hamiltonian $H: T^{*} Q \rightarrow R$ is defined to be

$$
H\left(\alpha_{q}\right)=\frac{1}{2} K\left(\left(K^{b}\right)^{-1}\left(\alpha_{q}\right),\left(K^{b}\right)^{-1}\left(\alpha_{q}\right)\right)+V \circ \tau^{*}\left(\alpha_{q}\right)
$$

where $\tau^{*}: T^{*} Q \rightarrow Q$ is the canonical cotangent projection. Letting $\Omega_{0}$ denote the canonical symplectic structure [1] on $\mathrm{T}^{*} \mathrm{Q}$, the hamiltonian dynamics on $T^{*} Q$ is given by the unique vector field $X_{H}$ on $T^{*} Q$ such that

$$
d H(Y)=\Omega_{0}\left(X_{H}, Y\right)
$$

for all vector field $Y$ on $T^{*} Q$.

Let $\Phi^{T^{*}}: G \times T^{*} Q \rightarrow T^{*} Q$ be the cotangent lift of the action $\Phi$. Denoting $\Phi_{g}^{T^{*}}(\cdot)=\Phi^{T^{*}}(g, \cdot)$, we have

$$
H \circ \Phi_{g}^{T^{*}}=H,
$$

i.e. the group $G$ is a symmetry group of the hamiltonian system $\left(T^{*} Q, \Omega_{0}, X_{H}\right)$.

The modern setting of Nother's theorem relating symmetry to the existence of integrals of motion is given by the concept of momentum mapping. Let $\mathcal{G}$ denote the Lie algebra of $G$ and $\mathcal{G}^{*}$ its dual. The map

$$
J: T^{*} Q \rightarrow \mathcal{G}^{*}
$$


given by

$$
J\left(\alpha_{q}\right) \cdot \xi=\alpha_{q} \cdot \xi_{Q}(q) \quad \forall \alpha_{q} \in T_{q}^{*} Q
$$

is $A d^{*}$-equivariant, where $\xi \in \mathcal{G}$ and $\xi_{Q}$ is the infinitesimal generator of $\Phi$ on $Q$ associated to $\xi$ (see $[1]$ corollary 4.2.11). $J$ is a momentum mapping and the G-invariance of the hamiltonian $H$ (Eq.(5.3)) implies that $J$ is an integral, i.e. it is conserved along trajectories of $X_{H}$.

Returning to four-bar linkages, we restrict attention for the moment to linkages of Grashof type, i.e. the condition $s+l<p+q$ holds. To fix the parameterization, we let $l_{1}=s$. We also restrict ourselves to a connected component of the configuration space. Then

$$
Q=R^{2} \times S^{1} \times S^{1}
$$

the kinetic energy metric is $K$ defined by

$$
K((v, w),(\tilde{v}, \tilde{w}))=\frac{1}{2} m<v, \tilde{v}>+\frac{1}{2}<w, \mathbf{J} \tilde{w}>
$$

(see Eq.(4.10) of the previous section). The group of rigid motions in the plane is the symmetry group:

$$
G=S^{1} \times R^{2}
$$

Denoting a point in $Q$ by $\left(\mathbf{r}_{c}, \theta_{0}, \theta_{1}\right)$, the action $\Phi$ of $G=S^{1} \times R^{2}$ on $Q$ is given by

$$
\Phi\left((\phi, \mathbf{r}),\left(\mathbf{r}_{c}, \theta_{0}, \theta_{1}\right)\right)=\left(\mathbf{r}+\mathbf{r}_{c}, \theta_{0}+\phi, \theta_{1}+\phi\right)
$$

For our purposes it is convenient to eliminate the effect of translations altogether by putting the inertial observer at the center of mass of the system, i.e. $\mathbf{r}_{c} \equiv \mathbf{0}$. In [22] this process is explained via symplectic reduction by the translation group $R^{2}$. The effect of taking this step is that now,

$$
Q=S^{1} \times S^{1}, \quad G=S^{1}
$$

and,

$$
\Phi\left(\phi,\left(\theta_{0}, \theta_{1}\right)\right)=\left(\theta_{0}+\phi, \theta_{1}+\phi\right) .
$$


The kinetic energy metric on $Q$ is

$$
K\left(w_{1}, w_{2}\right)=<w_{1}, \mathbf{J} w_{2}>
$$

for $w_{1}, w_{2} \in T_{q} Q$.

The hamiltonian is given by

$$
H=\frac{1}{2}<\mu, \mathbf{J}^{-1} \mu>
$$

for $\mu=K^{b}(w)$. Using the abstract formula (5.4) and the action (5.6), one can show that a momentum mapping for the action $\Phi$ is

$$
J(\theta, \mu)=\mu_{0}+\mu_{1}
$$

Of course, $\nu=\mu_{0}+\mu_{1}$ is conserved along trajectories of $X_{H}$ for the hamiltonian $H$ in (5.8) and it is simply the net angular momentum of the floating four bar linkage relative to an observer at the system center of mass.

The dynamical trajectories are confined to level set of the form $J^{-1}(\nu)$. The group $S^{1}$ viewed as the isotropy subgroup of the momentum value $\nu$, acts freely on $J^{-1}(\nu)$ and one gets the symplectically reduces dynamics $X_{H_{\nu}}$ on the reduced phase space $P_{\nu}=J^{-1}(\nu) / S^{1} \simeq S^{1} \times R^{1}$. We discuss this further in the next section.

\section{6. (REDUCED) DYNAMICS \& RELATIVE EQUILIBRIA}

As in [22] it is possible to Poisson-reduce the dynamics. We recall that given a symplectic manifold $(M, \omega)$, and a smooth, free, proper, symplectic action of Lie group $G$ on $M$, the canonical Poisson structure on $M$ defined by

$$
\{f, g\}_{M}=\omega\left(X_{f}, X_{g}\right) \quad \forall f, g \in C^{\infty}(M)
$$

descends to a Poisson structure on the quotient $P=M / G$. The latter is defined by

$$
\{\hat{f}, \hat{g}\}_{M / G} \circ \pi=\{\hat{f} \circ \pi, \hat{g} \circ \pi\}_{M}
$$


where $\hat{f}, \hat{g} \in C^{\infty}(M / G)$ and $\pi: M \rightarrow M / G$ is the canonical projection. If $H: M \rightarrow R$ is a $G$-invariant hamiltonian, it induces $\hat{H}: M / G \rightarrow R$ defined by $\hat{H} \circ \pi=H$. We refer to the dynamics (vector field) $X_{\hat{H}}$ defined by

$$
X_{\hat{H}}(\hat{f})=\{\hat{f}, \hat{H}\} \quad \forall \hat{f} \in C^{\infty}(M / G)
$$

as the Poisson reduction of the dynamics $X_{H}$.

In the present context, with $Q, K, H$ as in (5.5)-(5.8), the space $M=T^{*}\left(S^{1} \times S^{1}\right)$ with parameterization $\left(\theta_{0}, \theta_{1}, \mu_{0}, \mu_{1}\right)$ carries the Poisson structure,

$$
\{f, g\}=\sum_{i=0}^{1}\left(\frac{\partial f}{\partial \theta_{i}} \cdot \frac{\partial g}{\partial \mu_{i}}-\frac{\partial f}{\partial \mu_{i}} \cdot \frac{\partial g}{\partial \theta_{i}}\right)
$$

for all $f, g \in C^{\infty}\left(T^{*}\left(S^{1} \times S^{1}\right)\right)$. The action of $G=S^{1}$ on $Q$ given by (5.6) is free and proper. The quotient $P=T^{*}\left(S^{1} \times S^{1}\right) / S^{1} \simeq S^{1} \times R^{2}$ carries a reduced Poisson structure. Parameterizing $P=T^{*}\left(S^{1} \times S^{1}\right) / S^{1}$ by $\theta_{10}=\left(\theta_{1}-\theta_{0}, \mu_{0}, \mu_{1}\right)$, the Poisson bracket on $P$ is given by,

$$
\{\hat{f}, \hat{g}\}_{T^{*}\left(S^{1} \times S^{1}\right) / S^{1}}=\frac{\partial \hat{f}}{\partial \theta_{10}} \cdot\left(\frac{\partial \hat{g}}{\partial \mu_{1}}-\frac{\partial \hat{g}}{\partial \mu_{0}}\right)-\frac{\partial \hat{g}}{\partial \theta_{10}} \cdot\left(\frac{\partial \hat{f}}{\partial \mu_{1}}-\frac{\partial \hat{f}}{\partial \mu_{0}}\right)
$$

which is a noncanonical structure. The reduced hamiltonian $\hat{H}$ is given by

$$
\hat{H}\left(\theta_{10}, \mu_{0}, \mu_{1}\right)=H\left(\theta_{0}, \theta_{1}, \mu_{0}, \mu_{1}\right)
$$

since the matrix $\mathbf{J}$ in (5.8) is a function of the difference $\theta_{10}=\theta_{1}-\theta_{0}$ only. The reduced dynamics is then immediately given:

$$
\begin{gathered}
\dot{\mu}_{0}=\frac{\partial \hat{H}}{\partial \theta_{10}} \\
\dot{\mu}_{1}=-\frac{\partial \hat{H}}{\partial \theta_{10}} \\
\dot{\theta}_{10}=\frac{\partial \hat{H}}{\partial \mu_{1}}-\frac{\partial \hat{H}}{\partial \mu_{0}} .
\end{gathered}
$$

Equation (6.6) involves complicated analytic expression resulting from the substitutions for $\theta_{3}$ and $\theta_{2}$ in terms of $\theta_{1}$ and $\theta_{1}$ as in [13]. Certain qualitative aspects of the 
reduced dynamics can still be explored, sidestepping analytic difficulties. For instance one can investigate relative equilibria.

Definition 6.1: $z_{e} \in M$ is a relative equilibrium for $X_{H}$ if

$$
X_{\hat{H}}\left(\pi\left(z_{e}\right)\right)=0
$$

Remark: Let $F_{X_{H}}^{t}$ be the flow of $X_{H}$ on $M$. Then $z_{e}$ is a relative equilibrium iff $F_{X_{H}}^{t}\left(z_{e}\right)$ is a stationary motion, i.e. there exists $\xi \in \mathcal{G}$ such that

$$
F_{X_{H}}^{t}\left(z_{e}\right)=\exp (t \xi)\left(z_{e}\right)
$$

Theorem 6.2: Let $J$ be an $A d^{*}$-equivariant momentum mapping on $M . z_{e} \in M$ is a relative equilibrium of $X_{H}$ iff there exists a $\xi \in \mathcal{G}$ such that $z_{e}$ is a critical point of

$$
H_{\xi}=H-\hat{J}(\xi)
$$

where $\hat{J}(\xi): M \rightarrow R: x \mapsto J(x)(\xi)$.

For proof, see chapter 4 of [1]. This theorem can be applied to a simple mechanical system with symmetry, $(Q, K, V, G)[19]$.

Theorem 6.3:(Smale) For simple mechanical system with symmetry $(Q, K, V, G)$, define

$$
V_{\xi}: Q \rightarrow R: q \mapsto V(q)-\frac{1}{2} K\left(\xi_{Q}(q), \xi_{Q}(q)\right)
$$

for each $\xi \in \mathcal{G}$. Then $z_{e}=\left(q_{e}, p_{e}\right) \in T^{*} Q$ is a relative equilibrium iff $q_{e}$ is a critical point of $V_{\xi}$ for some $\xi \in \mathcal{G}$ and $p_{e}=K^{b}\left(\xi_{Q}\left(q_{e}\right)\right)$.

Remark: It can be shown that, for a given $\xi \in \mathcal{G}, V_{\xi}$ has the symmetry,

$$
V_{\xi}\left(\Phi_{g}(x)\right)=V_{\xi}(x)
$$

for all $g \in G_{\xi}:=\left\{g \in G \mid A d_{g} \xi=\xi\right\}$. If $G=S^{1}, G_{\xi}=G$ and action $\Phi$ is free and proper. Then $Q / G_{\xi}$ is a smooth manifold and $\pi_{\xi}: Q \rightarrow Q / G_{\xi}$ is a submersion. Thus $V_{\xi}$ induces a function $\hat{V}_{\xi}$ on $Q / G_{\xi}$ such that

$$
V_{\xi}=\hat{V}_{\xi} \circ \pi_{\xi}
$$


Recalling that $V=0$, this theorem can be directly applied to the floating four-bar linkage system. Rewriting the kinetic energy given in section 4 by setting the origin of inertial space at the system of center of mass, we have

$$
T=\frac{1}{2}\langle\omega, \mathbf{J} \omega\rangle
$$

where $\omega=\left(\omega_{0}, \omega_{1}\right)$. It can be shown that the infinitesimal generator on $Q=S^{1} \times S^{1}$ is $\xi_{Q}(q)=(1,1)^{T}[21]$. Hence, by theorem $6.3,\left(\theta^{*}, \mu^{*}\right)$ is a relative equilibrium point on $T^{*} Q$ iff $\theta^{*}$ is a critical point of function

$$
V_{\xi}\left(\theta_{0}, \theta_{1}\right)=-(1,1) \mathbf{J}\left(\begin{array}{l}
1 \\
1
\end{array}\right)
$$

Applying Proposition 4.2 and the definition of $\tilde{\mathbf{J}}$ given in Theorem 4.1, we have

$$
V_{\xi}\left(\theta_{0}, \theta_{1}\right)=-\mathbf{e}^{T} \tilde{\mathbf{J}} \mathbf{e}
$$

where $\mathbf{e}=\left(\begin{array}{llll}1 & 1 & 1 & 1\end{array}\right)^{T}$. From Eq.(6.12), we observe that the diagonal terms, which include the inertia of bars, of matrix $\tilde{\mathbf{J}}$ do not effect the positions of critical points of function $V_{\xi}$. Since the elements of matrix $\tilde{\mathbf{J}}$ are only functions of $\theta_{1}-\theta_{0}$, above $V_{\xi}$ satisfies (6.10) for all $g \in S^{1}$. It follows that $\hat{V}_{\xi}\left(\theta_{10}\right)=V_{\xi}\left(\theta_{0}, \theta_{1}\right)$. Then, the critical points of $V_{\xi},\left(\theta_{0}^{*}, \theta_{1}^{*}\right)$, will make $\left(\theta_{1}^{*}-\theta_{0}^{*}\right)$ to be the critical points of $\hat{V}_{\xi}$. At relative equilibrium, the relative angles between bars are fixed and the whole system rotates around the system center of mass with constant angular velocity. Unlike the planar two-body case, the relative equilibrium shapes depend on the values of the masses and the lengths of bars. From the expression of matrices $\tilde{\mathbf{J}}$ and the relations between relative angles given in [13], it is very difficult to find critical points of function $\hat{V}_{\xi}$ analytically. However, numerically searching for critical points is easy since now $\hat{V}_{\xi}$ is only a function of one variable. In the following, we give an example to computing the relative equilibria by applying above theorem.

\section{Example:}

An assembly in Fig. 2 is a possible structure of a robot arm. The parameters are given as follows

$$
m_{0}=3, \quad m_{1}=1, \quad m_{2}=30, \quad m_{3}=1 ;
$$




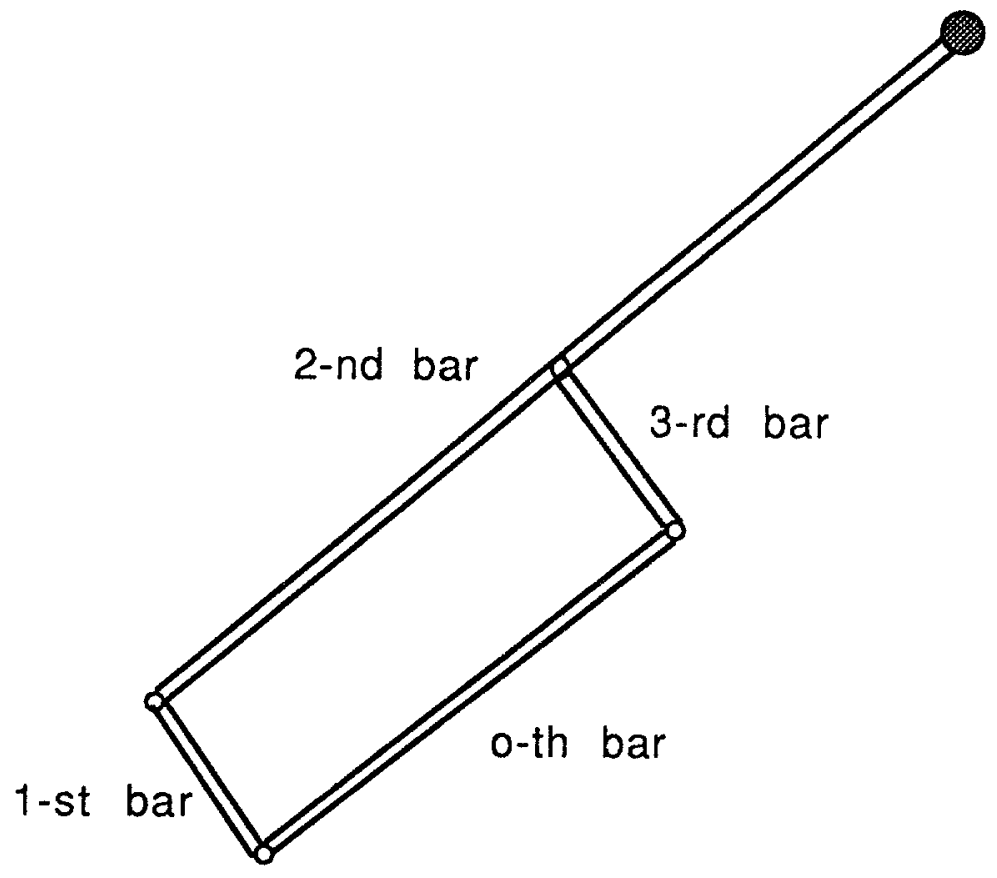

Fig. 2

$$
\begin{aligned}
\mathrm{d}(0,3)=(-1.5,0), & \mathrm{d}(0,1)=(1.5,0) \\
\mathrm{d}(1,0)=(-0.5,0), & \mathrm{d}(1,2)=(0.5,0) \\
\mathrm{d}(2,1)=(-6,0), & \mathrm{d}(2,3)=(-3,0) \\
\mathrm{d}(3,2)=(-0.55,0), & \mathrm{d}(3,0)=(0.55,0)
\end{aligned}
$$

Thus, the lengths of links are

$$
l_{0}=3, \quad l_{1}=1, \quad l_{2}=3, \quad l_{3}=1.1
$$

It is clear that $s+l<p+q$ and $s=l_{1}$ are satisfied.

The graph of $\hat{V}_{\xi}$ is given in Fig. 3. From this figure we can see two critical points appear at $\theta_{10}=7 \mathrm{deg}$ and $\theta_{10}=186 \mathrm{deg}$. The shape determined by these two angles are shown in Fig. 4. 


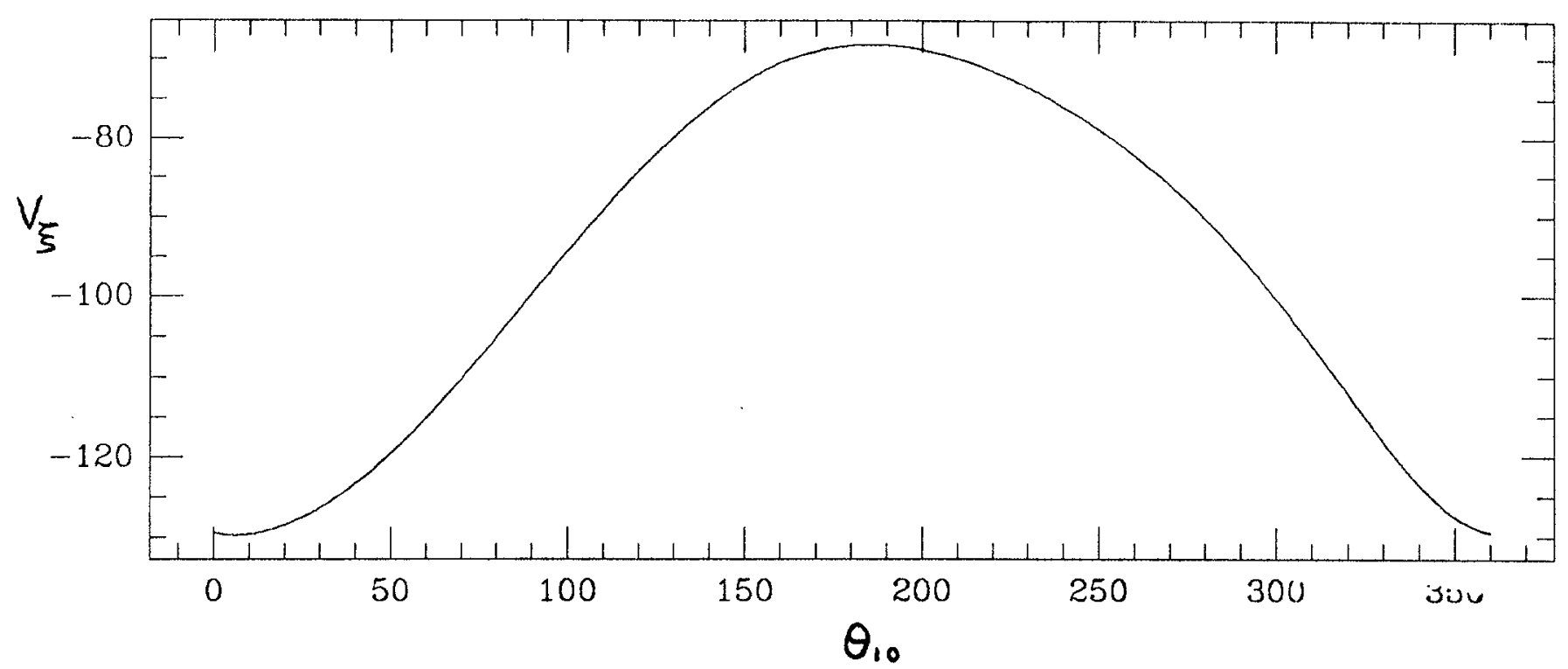

Fig. 3

\section{CONCLUSIONS}

In this paper, we gave sufficient conditions which make the configuration space of a closed four-bar mechanism be a smooth manifold by applying the classical theory of a mechanisms due to Grashof. Under one of them, i.e. $s+l<p+q$ we derived well defined expression of kinetic energy, or Lagrangian. This Lagrangian is invariant under the action of $S E(2)$, the rigid motion group in plane. It turns out that the four-bar mechanism is a simple mechanical system with symmetry. Applying Poisson reduction we obtained the reduced dynamics. Furthermore, by using Smale's theorem on relative equilibria we found a function whose critical points give the relative equilibria for our system. An example of its application was given.

\section{REFERENCES}

[1] Abraham, R. \& J. E. Marsden, Foundations of Mechanics (Second edition), Revised, Enlarged, Reset, Benjamin/Cummings, Reading, 1978. 

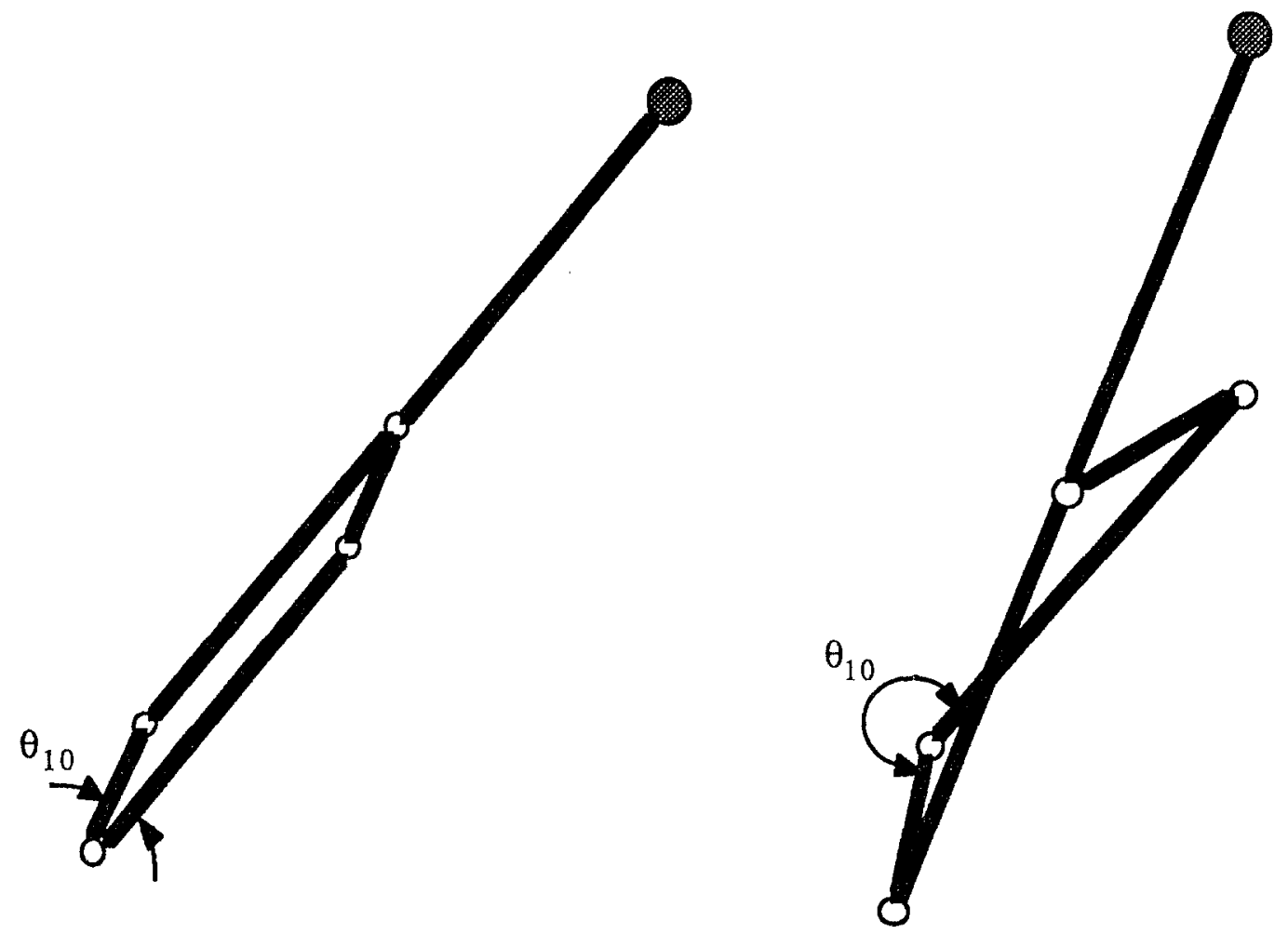

Fig. 4

[2] Baillieul, J., "Linearized Models for the Control of Rotating Beams," Proc. 27th IEEE Conf. Dec. Control, New York (1988).

[3] Baillieul, J. \& M. Levi, "Rotational Elastic Dynamics," Physica D, 27D (1987).

[4] Bianchi, G. \& W. Schielen, "Dynamics of Multibody Systems," Proceedings of IUTAM/IFToMM Symposium, Udine/Italy (1985).

[5] CIME, "Stereodynamics," edizione cremonese, Roma., 1972.

[6] Dirac, P. A. M., "Generalized Hamiltonian dynamics," Proc. Roy. Soc. A 246 (1958).

[7] Gibson, C. G. \& P. E. Newstead, "On the Geometry of the Planar 4-Bar Mechanism," Acta Applicandae Math. 7 (1986), 113-135.

[8] Grashof, F., Theoretische Maschinenlehre, Verlag L. Voss, Leipzig, 1883. 
[9] Grossman, R., P. S. Krishnaprasad \& J. E. Marsden, "The Dynamics of Two Coupled Three Dimensional Rigid Bodies," in Dynamical Systems Approaches to Nonlinear Problems in Systems and Circuits, F. Salam \& M. Levi, eds., Philadelphia, 1988., 1987, 373-378 \& SIAM Publ.,.

[10] Hunt, K. H., Kinematic Geometry of Mechanisms, Clarendon Press, Oxford, 1978.

[11] Krishnaprasad, P. S. \& J. E. Marsden, "Hamiltonian Structures \& Stability for Rigid Bodies with Flexible Attachments," Arch. Rat. Mech. Anal. 98 (1987).

[12] Oh, Y. G., N. Sreenath, P. S. Krishnaprasad \& J. E. Marsden, "The Dynamics of Coupled Planar Rigid Bodies Part II: Bifurcation, Periodic Orbits, and Chaos," J. Dynamics \& Differential Equations 1(1989), 269-298.

[13] Paul, B., Kinematics and Dynamics of Planar Machinery, Prentice-Hall, Inc., 1979.

[14] - "A Reassessment of Grashof's Criterion," Tran. of the ASME, J. of Mech. Design Jul 101 (1979), 515-518.

[15] Posbergh, T., "Modeling and Control of Mixed and Flexible Structures," University of Maryland, College Park, Ph.D. Thesis, 1988, also, Systems Research Center Technical Report SRC TR88-58.

[16] Posbergh, T., P. S. Krishnaprasad \& J. E. Marsden, "Differential Geometry : The Interface between Pure and Applied Mathematics," 1987.

[17] Simo, J. C., J. E. Marsden \& P. S. Krishnaprasad, "The Hamiltonian Structure of Nonlinear Elasticity: The Material and Convective Representation of Rods, Plates and Shells," Arch. Rat. Mech. \& Anal. 104 (1988), 125-183.

[18] Simo, J. C., T. Posbergh \& J. E. Marsden, Nonlinear Stability of Geometrically Exact Rods by the Energy-Momentum Method, Stanford University, Division of Applied Mechanics., 1989, preprint.

[19] Smale, S., "Topology and Mechanics, I, II," Invent. Math.11(1970), 45-64.

[20] Sniatycki, J., "Dirac Brackets in Geometric Dynamics," in Ann. Inst. Henri Poincare XX \#4, 1974, 365-372. 
[21] Sreenath, N., "Modeling and Control of Multibody Systems," University of Maryland, College Park, Ph.D. Thesis, 1987, also, Systems Research Center Technical Report SRC TR87-163.

[22] Sreenath, N., Y. G. Oh, P. S. Krishnaprasad \& J. E. M. (1988),, "The Dynamics of Coupled Planar Rigid Bodies Part I: Reduction, Equilibria \& Stability," in Dynamics \& Stability of Systems 3 \#1\&2, 25-49.

[23] Wang, L-S. \& P. S. Krishnaprasad, "Relative Equilibria of Two Rigid Bodies connected by a Ball-in-Socket Joint," IEEE Conference on Decision and Control (Dec. 1989.).

[24] Wittenburg, J., Dynamics of Multibody Systems, B.G. Teubner, Stuttgart, 1977. 